\title{
B. Relations entre la Suisse et les pays en développement et en transition
}

Gérard Perroulaz et Xavier Tschumi Canosa

\section{(2) OpenEdition}

Édition électronique

URL : http://journals.openedition.org/aspd/438

DOI : 10.4000/aspd.438

ISSN : 1663-9669

Éditeur

Institut de hautes études internationales et du développement

\section{Édition imprimée}

Date de publication : 1 avril 2005

Pagination : 220-229

ISSN : 1660-5934

\section{Référence électronique}

Gérard Perroulaz et Xavier Tschumi Canosa, «B. Relations entre la Suisse et les pays en

développement et en transition ", Annuaire suisse de politique de développement [En ligne], 24-1 | 2005, mis en ligne le 05 mars 2010, consulté le 08 septembre 2020. URL : http://journals.openedition.org/ aspd/438; DOI : https://doi.org/10.4000/aspd.438 
Tableau 1 ${ }^{\mathrm{a}}$ : Synthèse des relations de la Suisse avec les pays en développement et les pays en transition ${ }^{\mathrm{b}}$ en 2003 (en milliers de francs et en pourcetage)

\begin{tabular}{|c|c|c|c|c|c|c|c|}
\hline & $A P(D), A^{A S P}$ & Aide privée $^{d}$ & GRE $^{\mathrm{e}}$ & IDE $^{f}$ & Importations & Exportations & $\begin{array}{r}\text { Solde } \\
\text { commercial }{ }^{\mathrm{g}} \\
\end{array}$ \\
\hline \multicolumn{8}{|l|}{ En milliers de francs } \\
\hline Pays en développement & 1'270'675 & $376 ' 874$ & 99'107 & 2'758'300 & 8'197'274 & $15^{\prime} 288^{\prime} 303$ & 7'091'029 \\
\hline Pays les moins avancés (PMA) & $341 ' 377$ & $151 ' 722$ & $-1 ' 786$ & $21 ' 500$ & $158 ' 587$ & 316'146.0 & $157 ' 559$ \\
\hline Pays à faible revenu (hors PMA) & $176 ' 443$ & $52 ' 822$ & $-44^{\prime} 800$ & $192^{\prime} 800$ & 1'326'900 & 1'773'680.0 & $446 ' 780$ \\
\hline Pays à revenu moyen & $300 ' 787$ & $104 ' 840$ & $163 ' 285$ & 2'532'600 & 6'699'552 & 13'082'770.0 & 6'383'218 \\
\hline Pays à revenu plus élevé & & & $-17 ' 592$ & $-9^{\prime} 100$ & $12 ' 235$ & $115^{\prime} 707.0$ & $103^{\prime} 472$ \\
\hline Projets régionaux et non ventilé & 452 '068 & $67 ' 490$ & & $20 ' 500$ & & & 0 \\
\hline Pays en transition & $90 ' 946$ & $15^{\prime} 443$ & $-17 ' 857$ & 1'560'800 & 7'536'704 & $15^{\prime} 795^{\prime} 709$ & 8'259'005 \\
\hline Pays en développement plus avancés & 1'448 & 1'853 & 4'974 & $108^{\prime} 400$ & 3'632'946 & $10^{\prime} 493^{\prime} 908.0$ & 6'860'962 \\
\hline $\begin{array}{l}\text { Pays d'Europe centrale et orientale, } \\
\text { nouveaux Etats indépendants }\end{array}$ & $89 ' 458$ & $13^{\prime} 591$ & $-22 ' 831$ & 1'452'400 & 3'903'758 & 5'301'801.0 & 1'398'043 \\
\hline Non ventilé et projets régionaux & 40 & & & .. & & & 0 \\
\hline
\end{tabular}

\section{En pourcentage}

Pays en développement

Pays les moins avancés (PMA)

Pays à faible revenu (hors PMA)

Pays à revenu moyen

Pays à revenu plus élevé

Projets régionaux et non ventilé

(2)

Pays en transition

Pays en développement plus avancés

Pays d'Europe centrale et orientale

nouveaux Etats indépendants

$\begin{array}{rr}26.9 & 40.9 \\ 13.9 & 27.7 \\ 23.7 & 0.0 \\ 35.6 & 17.4\end{array}$

$40.3-n$

n.a. 0.8

$0.8-1.9$

921

$\begin{array}{rrrrr}40.3 & \text { n.a. } & 0.8 & 1.9 & 2.1 \\ 14.0 & \text { n.a. } & 7.0 & 16.2 & 11.6 \\ 27.8 & \text { n.a. } & 91.8 & 81.7 & 85.6\end{array}$

\begin{tabular}{rrr}
0.8 & n.a. & 91.8 \\
0.0 & n.a. & -0.3 \\
\hline
\end{tabular}

0.3

$\begin{array}{rr}0.1 & 85.6 \\ --1\end{array}$

Non ventilé et projets régionaux

Sources: Aide suisse aux pays en développement et aux pays en transition 2003, DDC, 2005; Direction générale des douanes, Statistique annuelle du commerce extérieur de la Suisse 2003, Berne, 2004.

\begin{tabular}{rrrrrr}
1.6 & 12.0 & n.a. & 6.9 & 48.2 & 66.4 \\
98.4 & 88.0 & n.a. & 93.1 & 51.8 & 33.6 \\
\hline- & - & - & - & - & -
\end{tabular}


n.a. = non applicable

.. = non disponible

a Le tableau 1 correspond au tableau 4.1. dans les Annuaires 2002 à 2004

${ }^{b}$ Les niveaux de revenu correspondent à la classification du CAD, qui figure à la fin de cet Annuaire.

Le CAD distingue les PMA, les autres pays à faible revenu (RNB par habitant inférieur à 745 dollars en 2001), les pays à revenu intermédiaire (RNB entre 746 et 9205 dollars par habitant), et les pays à revenu élevé (uniquement Bahreïn). Les pays et territoires en transition sont dans une catégorie à part, dont l'aide est comprise non pas dans I'APD, mais dans un agrégat séparé d'aide publique.

c $\mathrm{AP}(\mathrm{D})=$ aide publique (au développement), $\mathrm{AASP}=$ autres apports du secteur public.

d Aide privée = aide privée des ONG (projets financés par les fonds propres des ONG sans les contributions publiques).

e GRE = garantie contre les risques à l'exportation. Les chiffres négatifs dans les Garanties contre les risques à l'exportation (GRE) correspondent à une diminution du montant de la garantie.

IDE = investissements directs à l'étranger. Pour les investissement directs à l'étranger, les données fournies par la Banque nationale suisse sont partielles: on n'a pas les données pour tous les pays, ni les sous-totaux par région et catégorie de pays. Pour des raisons de confidentialité, certains montants d'investissements dans quelques pays ne sont pas montrés de manière isolée, mais uniquement dans les totaux. Des chiffres négatifs pour les investissements directs correspondent à des désinvestissements dans un pays (flux de capitaux en retour).

${ }^{9}$ Solde commercial: un solde positif montre que les exportations vers un pays ou une région sont supérieures aux importations en provenance de ce pays ou de cette région. Un solde négatif montre que les importations en provenance d'un pays ou d'une région sont supérieures aux exportations de la Suisse vers ce pays ou cette région. 
Tableau 2a: Relations de la Suisse avec les pays en développement ${ }^{\mathrm{b}}$ (partie I de la liste du CAD) en 2003, en milliers de francs

\begin{tabular}{|c|c|c|c|c|c|c|c|}
\hline & APD, AASPc & Aide privée $^{d}$ & $\mathbf{G R E}^{\mathrm{e}}$ & IDE $^{\dagger}$ & Importations & Exportations & $\begin{array}{r}\text { Solde } \\
\text { commercial }^{\mathrm{g}} \\
\end{array}$ \\
\hline Total & 1'270'675 & $376{ }^{\prime} 874$ & 99'107 & 2'758'300 & 8'197'274 & $15^{\prime} 288^{\prime} 303$ & 7'091'029 \\
\hline Europe & $135 ' 579$ & $17^{\prime} 493$ & $111^{\prime} 793$ & $243^{\prime} 200$ & $508^{\prime} 123$ & $2^{\prime} 160 ' 986$ & 1'652'863 \\
\hline Pays à faible revenu (hors PMA) & 4'395 & 36 & 0 & 0 & $1 ' 064$ & $18^{\prime} 157$ & $17^{\prime} 093$ \\
\hline Moldova & 4 4'395 & 36 & & .. & 1'064 & $18^{\prime} 157$ & $17^{\prime} 093$ \\
\hline Pays à revenu moyen & $121^{\prime} 486$ & $16 ' 086$ & $111^{\prime} 793$ & $100 ' 300$ & $507 ' 059$ & 2'142'829 & 1'635'770 \\
\hline Albanie & $14^{\prime} 235$ & 3'761 & & .. & 739 & $17^{\prime} 801$ & $17^{\prime} 062$ \\
\hline Bosnie-Herzégovine & $18^{\prime} 889$ & 4'196 & & .. & $6^{\prime} 163$ & $36^{\prime} 554$ & $30 ' 391$ \\
\hline Croatie & $1 ' 878$ & 25 & $-1 ' 517$ & $63^{\prime} 200$ & $44^{\prime} 157$ & $213^{\prime} 732$ & $169^{\prime} 575$ \\
\hline Etats ex-Yougoslavie, non spécifié & 146 & 4'956 & & .. & & & 0 \\
\hline Macédoine (ERYM) & $6 \prime 596$ & 422 & & .. & $5^{\prime} 172$ & $31^{\prime} 642$ & $26^{\prime} 470$ \\
\hline Serbie-et-Monténégro & 77'362 & $2^{\prime} 079$ & & .. & $18^{\prime} 268$ & $192 ' 872$ & $174 ' 604$ \\
\hline Turquie & 2'380 & 647 & $113^{\prime} 310$ & $37^{\prime} 100$ & $432 ' 560$ & $1^{\prime} 650^{\prime} 228$ & $1^{\prime \prime} 217^{\prime} 668$ \\
\hline Non ventilé et projets régionaux & 9'698 & $1 ' 370$ & &.. & & & \\
\hline Afrique & $344^{\prime} 146$ & $125^{\prime} 129$ & -655 & $-69^{\prime} 300$ & 1'658'770 & 1'849'880 & $191^{\prime} 110$ \\
\hline Pays les moins avancés (PMA) & $264 ' 828$ & $102 ' 801$ & $-1 ' 786$ & 0 & $58 ' 785$ & $194 ' 321$ & $135^{\prime} 536$ \\
\hline Angola & $9^{\prime} 223$ & 8'152 & $-1 ' 786$ & .. & 213 & $7^{\prime} 665$ & $7 ' 452$ \\
\hline Bénin & $9^{\prime} 454$ & 3'973 & & .. & 45 & $21^{\prime} 227$ & $21^{\prime} 182$ \\
\hline Burkina Faso & $27^{\prime} 740$ & 4'320 & & ... & $1^{\prime} 050$ & $1^{\prime} 604$ & 554 \\
\hline Burundi & 4'410 & 1'917 & & .. & $2^{\prime} 248$ & 556 & $-1^{\prime} 692$ \\
\hline Cap-Vert & $3^{\prime} 493$ & & & ... & 14 & 472 & 458 \\
\hline Centrafricaine, Rép. & 167 & $1 ' 201$ & & .. & 52 & 94 & 42 \\
\hline Comores & 100 & 75 & & .. & 134 & 98 & -36 \\
\hline Congo, Rép. dém. & $46^{\prime} 705$ & $9^{\prime} 447$ & &.. & 3'105 & 4'725 & 1'620 \\
\hline Djibouti & 0 & 0 & & .. & 6 & 542 & 536 \\
\hline Erythrée & $2^{\prime} 439$ & 1'054 & & .. & 588 & 1'149 & 561 \\
\hline Ethiopie & 7'073 & $10^{\prime} 269$ & & ... & $3^{\prime} 661$ & 8'301 & $4^{\prime} 640$ \\
\hline Gambie & 1 & 90 & & .. & 17 & $1 ' 049$ & $1 ' 032$ \\
\hline Guinée & 731 & $1 ' 665$ & & .. & 882 & $25^{\prime} 095$ & $24^{\prime} 213$ \\
\hline
\end{tabular}




\begin{tabular}{|c|c|c|c|c|c|c|c|}
\hline & APD, AASPc & Aide privée $^{d}$ & GRE & IDE $^{f}$ & Importations & Exportations & $\begin{array}{r}\text { Solde } \\
\text { commercial }\end{array}$ \\
\hline \multicolumn{8}{|l|}{ Afrique (suite) } \\
\hline Guinée équatoriale & 66 & 1'990 & & ... & 541 & 496 & -45 \\
\hline Guinée-Bissau & 1'269 & 282 & & ... & 0 & 112 & 112 \\
\hline Lesotho & 1'118 & 876 & & .. & 0 & $1 ' 219$ & $1 ' 219$ \\
\hline Liberia & 3'797 & 1'674 & & .. & 94 & $10^{\prime} 355$ & $10^{\prime} 261$ \\
\hline Madagascar & 6'945 & 2'533 & & .. & $5 ' 159$ & $3^{\prime} 281$ & $-1^{\prime} 878$ \\
\hline Malawi & 196 & 970 & & .. & $9^{\prime} 825$ & 681 & $-9^{\prime} 144$ \\
\hline Mali & $11^{\prime} 822$ & $6^{\prime} 714$ & & .. & $1 ' 096$ & 1'540 & 444 \\
\hline Mauritanie & $1 ' 086$ & 1'879 & & .. & 90 & 1'897 & $1 ' 807$ \\
\hline Mayotte & & & & .. & 0 & 4 & 4 \\
\hline Mozambique & $28^{\prime} 130$ & $7^{\prime} 062$ & & .. & 2'161 & 1'865 & -296 \\
\hline Niger & $10^{\prime} 049$ & $1 ' 296$ & & .. & $1 ' 402$ & $13^{\prime} 990$ & $12^{\prime} 588$ \\
\hline Ouganda & 2'183 & 3'982 & & .. & $13^{\prime} 820$ & 5'182 & $-8^{\prime} 638$ \\
\hline Rwanda & 9'069 & $2 ' 415$ & & .. & 41 & 1'850 & 1'809 \\
\hline São Tomé et Príncipe & & 2 & & .. & 126 & 639 & 513 \\
\hline Sénégal & $4 ' 290$ & $1 ' 704$ & & .. & $2^{\prime} 826$ & $16^{\prime} 903$ & $14^{\prime} 077$ \\
\hline Sierra Leone & $12^{\prime} 855$ & 27 & & .. & 39 & 687 & 648 \\
\hline Somalie & 923 & $2 ' 705$ & & .. & 172 & 127 & -45 \\
\hline Soudan & $10^{\prime} 110$ & $12^{\prime} 615$ & & .. & 718 & $30^{\prime} 273$ & $29^{\prime} 555$ \\
\hline Sainte-Hélène & & & & ... & 5 & 4 & -1 \\
\hline Tanzanie & $34^{\prime} 446$ & $6^{\prime} 412$ & & .. & $5^{\prime} 423$ & $8 ' 550$ & $3 \prime 127$ \\
\hline Tchad & $13^{\prime} 012$ & $1 ' 922$ & & .. & 340 & 678 & 338 \\
\hline Togo & $1 ' 023$ & 933 & & .. & $1 ' 250$ & $16^{\prime} 955$ & $15^{\prime} 705$ \\
\hline Zambie & 904 & $2 ' 645$ & & .. & $1 ' 642$ & 4'456 & $2^{\prime} 814$ \\
\hline Pays à faible revenu (hors PMA) & $19^{\prime} 470$ & $13^{\prime} 021$ & $-1 ' 250$ & .. & $458 ' 215$ & $263^{\prime} 787$ & $-194 ' 428$ \\
\hline Cameroun & 4,020 & $5 ' 857$ & &.. & 2'985 & $11^{\prime} 618$ & $8^{\prime} 633$ \\
\hline Congo, Rép. & 25 & 532 & & .. & 171 & $13^{\prime} 603$ & $13^{\prime} 432$ \\
\hline Côte d'Ivoire & 2'467 & 326 & -587 & $3 ' 700$ & 21'552 & $29^{\prime} 982$ & $8^{\prime} 430$ \\
\hline Ghana & $10^{\prime} 489$ & 877 & & .. & $44^{\prime} 920$ & $16^{\prime} 204$ & $-28^{\prime} 716$ \\
\hline Kenya & 1'325 & 3'093 & -671 & .. & 19'339 & $27^{\prime} 684$ & 8'345 \\
\hline Nigeria & 382 & 257 & & $25^{\prime} 800$ & $351^{\prime} 774$ & $140 ' 545$ & $-211^{\prime} 229$ \\
\hline Zimbabwe & 762 & $2^{\prime} 080$ & 8 & ... & $17^{\prime} 474$ & $24 ' 151$ & $6^{\prime} 677$ \\
\hline
\end{tabular}




\begin{tabular}{|c|c|c|c|c|c|c|c|}
\hline & APD, AASPc & Aide privée $^{d}$ & $\mathbf{G R E}^{\mathrm{e}}$ & IDE $^{\mathrm{f}}$ & Importations & Exportations & $\begin{array}{r}\text { Solde } \\
\text { commercial }^{g}\end{array}$ \\
\hline \multicolumn{8}{|l|}{ Afrique (suite) } \\
\hline Pays à revenu moyen & $24^{\prime} 768$ & 7'099 & 2'381 & .. & 1'141'770 & 1'391'772 & $250 ' 002$ \\
\hline Afrique du Sud & $10^{\prime} 566$ & $2^{\prime} 870$ & $-1^{\prime} 020$ & ... & $862^{\prime} 226$ & $523^{\prime} 026$ & $-339^{\prime} 200$ \\
\hline Algérie & $2^{\prime} 802$ & 704 & $-17^{\prime} 523$ & .. & $128^{\prime} 540$ & $140^{\prime} 069$ & $11^{\prime} 529$ \\
\hline Botswana & & 23 & &.. & 104 & $33^{\prime} 946$ & $33^{\prime} 842$ \\
\hline Egypte & $3^{\prime} 657$ & 1'620 & $-10^{\prime} 310$ & $31 ' 300$ & $21 ' 517$ & $415^{\prime} 100$ & $393 ' 583$ \\
\hline Gabon & 6 & 657 & & .. & 179 & $2^{\prime} 672$ & $2 ' 493$ \\
\hline Maroc & 4'589 & 639 & 960 & $33^{\prime} 600$ & $74^{\prime} 155$ & $144^{\prime} 649$ & $70^{\prime} 494$ \\
\hline Maurice & & 12 & -245 & .. & $28^{\prime} 977$ & $40^{\prime} 049$ & $11^{\prime} 072$ \\
\hline Namibie & $1 ' 075$ & 314 & & .. & 1'879 & $2^{\prime} 411$ & 532 \\
\hline Seychelles & & 11 & & .. & 101 & 1'701 & $1^{\prime} 600$ \\
\hline Swaziland & 100 & 29 & & .. & 137 & $1 ' 213$ & $1 ' 076$ \\
\hline Tunisie & $1^{\prime} 973$ & 220 & $30^{\prime} 519$ & .. & $23^{\prime} 955$ & $86^{\prime} 936$ & $62^{\prime} 981$ \\
\hline Non ventilé et projets régionaux & 35 '079 & $2 ' 208$ & & .. & & & \\
\hline Amérique & $136^{\prime} 025$ & $56 ' 281$ & $-68^{\prime} 883$ & $2^{\prime} 199 ' 200$ & 1'135'629 & $3^{\prime} 307^{\prime} 821$ & $2^{\prime} 172 ' 192$ \\
\hline Pays les moins avancés (PMA) & 4'121 & 3'940 & 0 & .. & 3'144 & $1 ' 418$ & $-1^{\prime} 726$ \\
\hline Haïti & $4^{\prime} 121$ & $3^{\prime} 940$ & & .. & $3^{\prime} 144$ & $1 ' 418$ & $-1^{\prime} 726$ \\
\hline Pays à faible revenu (hors PMA) & $20 ' 084$ & 4'842 & 0 & .. & $2 ' 743$ & $2 ' 448$ & -295 \\
\hline Nicaragua & $200^{\prime} 084$ & $4^{\prime} 842$ & & .. & $2 ' 743$ & $2^{\prime} 448$ & -295 \\
\hline Pays à revenu moyen & $93^{\prime} 560$ & $46^{\prime} 213$ & $-68 ' 883$ & .. & 1'129'742 & 3'303'955 & $2^{\prime} 174^{\prime} 213$ \\
\hline Antigua-et-Barbuda & & & & .. & 890 & $3^{\prime} 675$ & 2'785 \\
\hline Anguilla & & & & .. & 4 & 68 & 64 \\
\hline Argentine & 438 & 535 & $-3^{\prime} 585$ & $356^{\prime} 800$ & $57^{\prime} 926$ & $248^{\prime} 842$ & $190 ' 916$ \\
\hline Barbade & & & & .. & 1'733 & 4'267 & 2'534 \\
\hline Bélize & & 22 & & .. & 20 & 620 & 600 \\
\hline Bolivie & $18^{\prime} 990$ & $5^{\prime} 431$ & $-1^{\prime} 099$ & .. & 1'595 & 7'848 & $6^{\prime} 253$ \\
\hline Brésil & $5^{\prime} 816$ & 8'159 & $-18^{\prime} 061$ & $162^{\prime} 100$ & $443^{\prime} 946$ & 1'163'573 & $719^{\prime} 627$ \\
\hline Chili & 1'666 & 786 & $-3^{\prime} 210$ & $-22^{\prime} 600$ & $53^{\prime} 728$ & $133^{\prime} 322$ & $79^{\prime} 594$ \\
\hline Colombie & $18^{\prime} 456$ & 5'558 & $-2^{\prime} 945$ & $37^{\prime} 300$ & $49^{\prime} 601$ & $187^{\prime} 148$ & $137 ' 547$ \\
\hline Costa Rica & 638 & 494 & & $86^{\prime} 000$ & $84^{\prime} 268$ & $31^{\prime} 870$ & -52 '398 \\
\hline Cuba & 4'358 & 849 & & & $28^{\prime} 828$ & 9'347 & $-19^{\prime} 481$ \\
\hline
\end{tabular}




\begin{tabular}{|c|c|c|c|c|c|c|c|}
\hline & APD, AASPc & Aide privée ${ }^{d}$ & $\mathbf{G R E}^{\mathrm{e}}$ & IDE $^{f}$ & Importations & Exportations & $\begin{array}{r}\text { Solde } \\
\text { commercial }^{9}\end{array}$ \\
\hline \multicolumn{8}{|l|}{ Amérique (suite) } \\
\hline Dominicaine, Rép. & $1 ' 167$ & 1'233 & $-1^{\prime} 106$ & ... & $18^{\prime} 760$ & $21^{\prime} 153$ & 2'393 \\
\hline Dominique & & & & .. & 60 & 1'598 & 1'538 \\
\hline El Salvador & $2 ' 465$ & 2'287 & -784 & .. & 2'839 & $11^{\prime} 121$ & $8^{\prime} 282$ \\
\hline Equateur & $10^{\prime} 128$ & $5^{\prime} 153$ & & $13^{\prime} 900$ & $43^{\prime} 804$ & $45^{\prime} 291$ & $1 ' 487$ \\
\hline Grenade & & & & .. & $2^{\prime} 008$ & 167 & $-1^{\prime} 841$ \\
\hline Guatemala & 3'752 & 4'831 & -669 & $-6^{\prime} 000$ & 16,009 & 24 '058 & $8^{\prime} 049$ \\
\hline Guyane & & & & ... & 198 & 1'087 & 889 \\
\hline Honduras & $2^{\prime} 663$ & 1'562 & & .. & $10^{\prime} 364$ & $18^{\prime} 446$ & $88^{\prime} 082$ \\
\hline Jamaïque & & & & .. & 3'314 & $15^{\prime} 973$ & $12^{\prime} 659$ \\
\hline Mexique & 428 & $2 ' 592$ & $-19^{\prime} 389$ & $28^{\prime} 700$ & $218^{\prime} 640$ & 914'042 & 695402 \\
\hline Montserrat & & & & ... & 0 & 63 & 63 \\
\hline Panama & & 30 & -353 & .. & $41 ' 395$ & $200^{\prime} 830$ & $159^{\prime} 435$ \\
\hline Paraguay & 771 & 678 & -44 & ... & $2 ' 345$ & $11^{\prime} 111$ & $8^{\prime} 766$ \\
\hline Pérou & $21 ' 341$ & $4^{\prime} 884$ & -498 & $-10^{\prime} 800$ & $19^{\prime} 250$ & $62^{\prime} 460$ & $43^{\prime} 210$ \\
\hline Saint-Vincent & & & & .. & 517 & 936 & 419 \\
\hline Sainte-Lucie & & & & .. & 37 & 1'909 & 1'872 \\
\hline Suriname & & & & .. & 145 & $2 ' 730$ & $2 ' 585$ \\
\hline Trinité et Tobago & & & & .. & 966 & 5'340 & $4 ' 374$ \\
\hline Turks et Caïques, îles & & & & .. & 84 & $1^{\prime} 004$ & 920 \\
\hline Uruguay & 333 & 116 & -247 & $37^{\prime} 200$ & $6 \prime 763$ & 80 '087 & 73'324 \\
\hline Venezuela & 148 & $1 ' 011$ & $-16^{\prime} 893$ & $176^{\prime} 600$ & $19^{\prime} 705$ & $93^{\prime} 969$ & $74^{\prime} 264$ \\
\hline Non ventilé et projets régionaux & $18 ' 259$ & $1 ' 286$ & & .. & & & \\
\hline Asie & $294^{\prime} 188$ & $115^{\prime} 512$ & $566^{\prime} 851$ & $378^{\prime} 800$ & $4^{\prime} 894^{\prime} 271$ & 7'966'972 & $3^{\prime} 072 ' 701$ \\
\hline Pays les moins avancés (PMA) & $72 ' 413$ & $44^{\prime} 971$ & 0 & 0 & $96 ' 654$ & $119^{\prime} 985$ & $23^{\prime} 331$ \\
\hline Afghanistan & $20^{\prime} 218$ & $10^{\prime} 943$ & & .. & 1'044 & 1'084 & 40 \\
\hline Bangladesh & $14^{\prime} 499$ & 3'345 & &.. & $65^{\prime} 164$ & $60^{\prime} 623$ & $-4^{\prime} 541$ \\
\hline Bhoutan & 7'573 & 842 & &.. & 0 & 643 & 643 \\
\hline Cambodge & 4'355 & $20 ' 257$ & & .. & $14^{\prime} 556$ & 6'694 & $-7 ' 862$ \\
\hline Laos & 4'058 & 916 & &.. & $3 ' 870$ & 713 & $-3^{\prime} 157$ \\
\hline Maldives & & & & .. & 208 & 2'157 & 1'949 \\
\hline
\end{tabular}


APD, AASPc Aide privée $^{d}$

GRE

IDE $^{\dagger} \quad$ Importations Exportations

Solde

Asie (suite)

\begin{tabular}{|c|c|c|c|c|c|c|c|}
\hline Myanmar (Birmanie) & 2'581 & 1'060 & & .. & $5^{\prime} 684$ & $3^{\prime} 069$ & $-2^{\prime} 615$ \\
\hline Népal & $19^{\prime} 129$ & $7^{\prime} 608$ & & .. & $5^{\prime} 786$ & $3^{\prime} 220$ & $-2^{\prime} 566$ \\
\hline Yémen & & & & .. & 342 & $41^{\prime} 782$ & $41^{\prime} 440$ \\
\hline Pays à faible revenu (hors PMA) & $132 ' 165$ & 34 '519 & $-43^{\prime} 550$ & $170 ' 400$ & 864 '504 & 1'488'630 & $624 ' 126$ \\
\hline Arménie & 2'963 & 651 & & .. & 1'545 & 8'296 & $6 ' 751$ \\
\hline Azerbaïdjan & 3'405 & 69 & $-2 ' 036$ & .. & 910 & $22^{\prime} 534$ & $21^{\prime} 624$ \\
\hline Corée, Dém. & 5'357 & 956 & &.. & 1'185 & 7'944 & $6 ' 759$ \\
\hline Géorgie & 9'310 & 300 & & .. & 499 & $7^{\prime} 607$ & 7'108 \\
\hline Inde & $33^{\prime} 155$ & $20^{\prime} 148$ & $-16^{\prime} 581$ & $135^{\prime} 400$ & $500^{\prime} 213$ & 741'633 & $241^{\prime} 420$ \\
\hline Indonésie & 5395 & 1'751 & $-22^{\prime} 720$ & $-12^{\prime} 900$ & $167^{\prime} 101$ & $289^{\prime} 367$ & $122 ' 266$ \\
\hline Kirghize, Rép. & $11 ' 571$ & 3'677 & & .. & 273 & 1'984 & 1'711 \\
\hline Mongolie & $2 ' 019$ & 704 & & .. & 1'750 & $6^{\prime} 299$ & 4'549 \\
\hline Ouzbékistan & 7'901 & 8 & &.. & 2'504 & $26^{\prime} 442$ & $23^{\prime} 938$ \\
\hline Pakistan & $17^{\prime} 861$ & 354 & $-2^{\prime} 130$ & $23^{\prime} 200$ & $36^{\prime} 647$ & $245^{\prime} 533$ & $208^{\prime} 886$ \\
\hline Tadjikistan & $18^{\prime} 035$ & 303 & &.. & 204 & 470 & 266 \\
\hline Timor-Oriental & 262 & 336 & & ... & 348 & 1'337 & 989 \\
\hline Vietnam & $14^{\prime} 930$ & $5^{\prime} 263$ & -83 & $24^{\prime} 700$ & $151 ' 325$ & $129^{\prime} 184$ & $-22^{\prime} 141$ \\
\hline Pays à revenu moyen & $60 ' 973$ & $35^{\prime} 424$ & $117^{\prime} 994$ & $163^{\prime} 600$ & 3'920'878 & 6'242'650 & 2'321'772 \\
\hline Arabie saoudite & & & & $-17^{\prime} 600$ & $173^{\prime} 786$ & 1'123'671 & $949 ' 885$ \\
\hline Chine & $16^{\prime} 785$ & 4'002 & $-22^{\prime} 282$ & 100 & 2'413'919 & $2^{\prime} 460^{\prime} 016$ & $46^{\prime} 097$ \\
\hline Irak & 7'504 & $5^{\prime} 638$ & -267 & .. & 0 & $38^{\prime} 406$ & $38^{\prime} 406$ \\
\hline Iran & 1'866 & 12 & $184 ' 539$ & .. & $170 ' 539$ & $541^{\prime} 480$ & $370^{\prime} 941$ \\
\hline Jordanie & 2'979 & 170 & $-2 ' 505$ & .. & $8^{\prime} 661$ & $124^{\prime} 104$ & $115^{\prime} 443$ \\
\hline Kazakhstan & 386 & $3 ' 425$ & $11^{\prime} 666$ &.. & $47^{\prime} 447$ & $77^{\prime} 716$ & $30^{\prime} 269$ \\
\hline Liban & 978 & 1'015 & 1'343 & $2^{\prime} 200$ & $190^{\prime} 825$ & $167 ' 589$ & $-23^{\prime} 236$ \\
\hline Malaisie & 347 & 298 & & $81^{\prime} 000$ & $174^{\prime} 749$ & $449^{\prime} 393$ & $274 ' 644$ \\
\hline Oman & & & & .. & 2'503 & $97 ' 811$ & $95 ' 308$ \\
\hline Philippines & 1'721 & 6'637 & $-10 ' 300$ & $-83^{\prime} 600$ & $89^{\prime} 200$ & $207^{\prime} 787$ & $118^{\prime} 587$ \\
\hline Sri Lanka & $6 ' 459$ & 1'997 & & .. & $37^{\prime} 051$ & $56^{\prime} 105$ & $19^{\prime} 054$ \\
\hline Syrie & 1'116 & 103 & & .. & 3'118 & $109^{\prime} 151$ & $106^{\prime} 033$ \\
\hline Thaïlande & $2^{\prime} 032$ & 466 & $-44^{\prime} 200$ & $181^{\prime} 500$ & $608^{\prime} 146$ & 777'595 & $169^{\prime} 449$ \\
\hline
\end{tabular}




\begin{tabular}{|c|c|c|c|c|c|c|c|}
\hline & APD, AASPc & Aide privée $^{d}$ & $\mathbf{G R E}^{\mathrm{e}}$ & $\mathrm{IDE}^{\mathrm{f}}$ & Importations & Exportations & $\begin{array}{r}\text { Solde } \\
\text { commercial }^{g}\end{array}$ \\
\hline \multicolumn{8}{|l|}{ Asie (suite) } \\
\hline Turkménistan & 1'050 & 50 & & .. & 573 & $6^{\prime} 515$ & $5^{\prime} 942$ \\
\hline Zones admin. palestinienne & $17^{\prime} 750$ & $11^{\prime} 610$ & & ... & 361 & 5'311 & 4'950 \\
\hline Pays à revenu plus élevé & & & $-17 ' 592$ & .. & $12 ' 235$ & $115^{\prime} 707$ & $103^{\prime} 472$ \\
\hline Bahreïn & & & $-17^{\prime} 592$ & .. & $12^{\prime} 235$ & $115^{\prime} 707$ & $103^{\prime} 472$ \\
\hline Non ventilé et projets régionaux & $28 ' 638$ & 598 & & ... & & & \\
\hline Océanie & 344 & 432 & 0 & 6'400 & 481 & $2^{\prime} 644$ & 2'163 \\
\hline Pays les moins avancés (PMA) & 15 & 11 & 0 & 0 & 4 & 422 & 418 \\
\hline Samoa & & 11 & & .. & 0 & 273 & 273 \\
\hline Salomon, îles & & & & .. & 3 & 47 & 44 \\
\hline Vanuatu & 15 & & & .. & 1 & 102 & 101 \\
\hline Pays à faible revenu (hors PMA) & 329 & 404 & 0 & 0 & 374 & 658 & 284 \\
\hline Papouasie-Nouvelle-Guinée & 329 & 404 & & .. & 374 & 658 & 284 \\
\hline Pays à revenu moyen & 0 & 18 & 0 & 0 & 103 & 1'564 & $1 ' 461$ \\
\hline Cook & & & & .. & 3 & 0 & -3 \\
\hline Fidji & & & & .. & 63 & $1 ' 426$ & 1'363 \\
\hline Micronésie & & 18 & & .. & 0 & 1 & 1 \\
\hline Nauru & & & & .. & 28 & 12 & -16 \\
\hline Niue & & & & .. & 9 & 0 & -9 \\
\hline Tonga & & & & .. & 0 & 125 & 125 \\
\hline Non ventilé et projets régionaux & & & & .. & & & \\
\hline Non ventilé par continent & 360 '394 & $62 ' 027$ & &.. & & & \\
\hline
\end{tabular}

Sources: Aide suisse aux pays en développement et aux pays en transition 2003, DDC, 2005; Direction générale des douanes, Statistique annuelle du commerce extérieur de la Suisse 2003, Berne, 2004.

.. = non disponible

a Le tableau 2 correspond au tableau 4.2. dans les Annuaires 2002 à 2004.

b Les niveaux de revenu correspondent à la classification du CAD, qui figure à la fin de cet Annuaire.

Le CAD distingue les PMA, les autres pays à faible revenu (RNB par habitant inférieur à 745 dollars en 2001), les pays à revenu intermédiaire (RNB entre 746 et 9205 dollars par habitant), et les pays à revenu élevé (uniquement Bahreïn).

$\mathrm{N} \quad \therefore$ APD = aide publique au développement, $\mathrm{AASP}=$ autres apports du secteur public

d Aide privée = aide privée des ONG (projets financés par les fonds propres des ONG sans les contributions publiques). 
e GRE = garantie contre les risques à l'exportation. Les chiffres négatifs dans les Garanties contre les risques à l'exportation (GRE) correspondent à une diminution du montant de la garantie.

${ }^{\dagger} \mathrm{IDE}=$ investissements directs à l'étranger. Pour les investissement directs à l'étranger, les données fournies par la Banque nationale suisse sont partielles: on n'a pas les données pour tous les pays, ni les sous-totaux par région et catégorie de pays. Pour des raisons de confidentialité, certains montants d'investissements dans quelques pays ne sont pas montrés de manière isolée, mais uniquement dans les totaux. Des chiffres négatifs pour les investissements directs correspondent à des désinvestissements dans un pays (flux de capitaux en retour).

g Solde commercial: un solde positif montre que les exportations vers un pays ou une région sont supérieures aux importations en provenance de ce pays ou de cette région. Un solde négatif montre que les importations en provenance d'un pays ou d'une région sont supérieures aux exportations de la Suisse vers ce pays ou cette région.

\section{Tableau 3 $3^{a}$ : Relations de la Suisse avec les pays en transition ${ }^{b}$ (partie II de la liste du CAD) en 2003, en milliers de francs}

\begin{tabular}{|c|c|c|c|c|c|c|c|}
\hline & AP, AASPc & Aide privée $^{d}$ & GRE $^{\mathrm{e}}$ & $\mathrm{IDE}^{\mathrm{f}}$ & Importations & Exportations & $\begin{array}{r}\text { Solde } \\
\text { commercial } 9 \\
\end{array}$ \\
\hline Total & $90 ' 946$ & $15^{\prime} 443$ & -17 '857 & 1'560'800 & 7'536'704 & $15^{\prime} 795^{\prime} 709$ & $8^{\prime} 259^{\prime} 005$ \\
\hline Pays en développement plus avancés (PED) & $1 ' 448$ & 1'853 & $-22^{\prime} 831$ & 1'452'400 & 3'632'946 & $10^{\prime} 493^{\prime} 908$ & $6^{\prime} 860 ' 962$ \\
\hline Antilles néerlandaises & & & & ... & 311 & $27 ' 898$ & $27 ' 587$ \\
\hline Aruba & & & & ... & 15 & $10 ' 833$ & $10^{\prime} 818$ \\
\hline Bahamas & & & & .. & $92 ' 522$ & 26 '075 & $-66^{\prime} 447$ \\
\hline Bermudes & & & & .. & 176 & $53^{\prime} 701$ & $53^{\prime} 525$ \\
\hline Brunei & & & & .. & 5 & $7^{\prime} 926$ & 7'921 \\
\hline Caïmans, Iles & & & & .. & 186 & $26 ' 117$ & $25^{\prime} 931$ \\
\hline Chypre & 5 & & & .. & 2 '355 & 80 '958 & $78^{\prime} 603$ \\
\hline Corée & 101 & 42 & -2 '852 & $26^{\prime} 500$ & $507 ' 748$ & 1'101'586 & $593 ' 838$ \\
\hline Emirats arabes unis & & & -705 & $-38^{\prime} 000$ & $128 ' 291$ & 908'461 & $780 ' 170$ \\
\hline Falkland, Iles & & & & ... & & 113 & 113 \\
\hline Gibraltar & & & & .. & $29^{\prime} 942$ & $11^{\prime} 444$ & $-18^{\prime} 498$ \\
\hline Hongkong, Chine & 30 & 6 & $-1 ' 049$ & $133^{\prime} 800$ & $648^{\prime} 720$ & 4'002'187 & 3'353'467 \\
\hline Israël & 569 & 1'523 & $-18 ' 225$ & .. & $370 ' 860$ & $626 ' 590$ & $255^{\prime} 730$ \\
\hline Koweït & 4 & & & .. & 406 & 244 '373 & $243^{\prime} 967$ \\
\hline Libye & 3 & & & .. & $755^{\prime} 881$ & $122 ' 304$ & $-633^{\prime} 577$ \\
\hline Macao & 7 & & & .. & 6'804 & 5'321 & $-1 ' 483$ \\
\hline Malte & 600 & & & .. & 4'782 & $77 ' 340$ & $72 ' 558$ \\
\hline Nouvelle-Calédonie & & & & .. & 19 & 968 & 949 \\
\hline Polynésie française & & & & .. & 906 & 3'203 & $2 ' 297$ \\
\hline Qatar & & & & .. & 228 & $116^{\prime} 953$ & $116^{\prime} 725$ \\
\hline Singapour & 2 & & & $-13^{\prime} 600$ & $228 ' 489$ & 1'358'677 & 1'130'188 \\
\hline
\end{tabular}




\begin{tabular}{|c|c|c|c|c|c|c|c|}
\hline & AP, AASPc & Aide privée $^{d}$ & $\mathbf{G R E}^{\mathrm{e}}$ & $\mathrm{IDE}^{\mathrm{f}}$ & Importations & Exportations & $\begin{array}{r}\text { Solde } \\
\text { commercial } 9\end{array}$ \\
\hline Slovénie & 76 & 151 & & 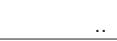 & 173 '034 & $292 ' 764$ & $119^{\prime} 730$ \\
\hline Taipei chinois (Taiwan) & 51 & 131 & & $41^{\prime} 100$ & $521 ' 027$ & 1'329'740 & $808^{\prime} 713$ \\
\hline Vierges, lles & & & & .. & $160 ' 239$ & $58 ' 376$ & $-101 ' 863$ \\
\hline PED plus avancés, non ventilé & & & & .. & & & 0 \\
\hline $\begin{array}{l}\text { Pays d'Europe centrale et orientale, } \\
\text { nouveaux Etats indépendants (PECO/NEI) }\end{array}$ & $89^{\prime} 458$ & $13^{\prime} 591$ & 4'974 & $108^{\prime} 400$ & 3'903'758 & 5'301'801 & 1'398'043 \\
\hline Bélarus & $2 ' 517$ & 352 & & .. & 3'732 & $27 ' 703$ & $23^{\prime} 971$ \\
\hline Bulgarie & $13^{\prime} 421$ & 829 & & .. & $61 ' 886$ & $178 ' 897$ & $117^{\prime} 011$ \\
\hline Estonie & 101 & 15 & & .. & $33^{\prime} 134$ & $43^{\prime} 178$ & $10^{\prime} 044$ \\
\hline Hongrie & 170 & 569 & & $189 ' 600$ & 711'199 & $846 ' 763$ & $135 ' 564$ \\
\hline Lettonie & 115 & 17 & $-1 ' 266$ & ... & $7 ' 766$ & $103 ' 655$ & $95 ' 889$ \\
\hline Lituanie & 604 & & -3 & .. & 27 '931 & $93 ' 861$ & $65 ' 930$ \\
\hline Pologne & 310 & 640 & $-10 ' 575$ & $124^{\prime} 700$ & $504 ' 620$ & 1'122'883 & $618 ' 263$ \\
\hline Roumanie & 20 '994 & 3'182 & $-10^{\prime} 033$ & & $142 ' 231$ & 305'191 & $162 ' 960$ \\
\hline Russie & $17 ' 568$ & 1'982 & 36 '009 & $107 ' 700$ & 1'175'563 & 1'064'728 & $-110^{\prime} 835$ \\
\hline Slovaquie & 809 & 75 & $-9^{\prime} 160$ & $-4^{\prime} 900$ & $306 ' 582$ & $283^{\prime} 417$ & $-23^{\prime} 165$ \\
\hline Tchèque, Rép. & 550 & 2'182 & & $-501^{\prime} 400$ & $882 ' 810$ & 1'032'420 & $149^{\prime} 610$ \\
\hline Ukraine & 7'408 & 1'159 & & ... & $46 ' 304$ & $199 ' 105$ & $152 ' 801$ \\
\hline PECO/NEI, non ventilé & 24 '893 & 2'590 & & .. & & & 0 \\
\hline Aide aux pays en transition, non ventilé & & & &.. & & & 0 \\
\hline
\end{tabular}

Sources: Aide suisse aux pays en développement et aux pays en transition 2003, DDC, 2005; Direction générale des douanes, Statistique annuelle du commerce extérieur de la Suisse 2003, Berne, 2004.

..$=$ non disponible

a Le tableau 3 correspond au tableau 4.3. dans les Annuaires 2002 à 2004.

${ }^{b}$ Les niveaux de revenu correspondent à la classification du CAD, qui figure à la fin de cet Annuaire.

Les pays et territoires en transition sont dans une catégorie à part, dont l'aide est comprise non pas dans I'APD, mais dans un agrégat séparé d'aide publique.

${ }^{c} \mathrm{AP}=$ aide publique, $\mathrm{AASP}=$ autres apports du secteur public.

${ }^{d}$ Aide privée = aide privée des ONG (projets financés par les fonds propres des ONG sans les contributions publiques).

e GRE = garantie contre les risques à l'exportation. Les chiffres négatifs dans les Garanties contre les risques à l'exportation (GRE) correspondent à une diminution du montant de la garantie.

${ }^{\dagger} \mathrm{IDE}=$ investissements directs à l'étranger. Pour les investissement directs à l'étranger, les données fournies par la Banque nationale suisse sont partielles: on n'a pas les données pour tous les pays, ni les sous-totaux par région et catégorie de pays. Pour des raisons de confidentialité, certains montants d'investissements dans quelques pays ne sont pas montrés de manière isolée, mais uniquement dans les totaux. Des chiffres négatifs pour les investissements directs correspondent à des désinvestissements dans un pays (flux de capitaux en retour).

Solde commercial: un solde positif montre que les exportations vers un pays ou une région sont supérieures aux importations en provenance de ce pays ou de cette région. Un solde négatif montre que les importations en provenance d'un pays ou d'une région sont supérieures aux exportations de la Suisse vers ce pays ou cette région. 